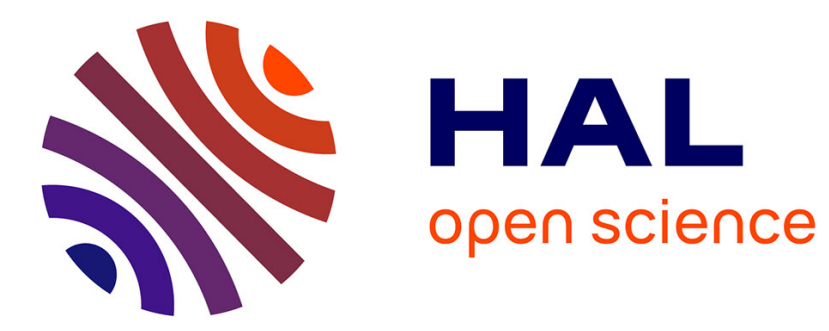

\title{
The Maxwell-Stefan diffusion limit for a kinetic model of mixtures
}

\author{
Laurent Boudin, Bérénice Grec, Francesco Salvarani
}

\section{To cite this version:}

Laurent Boudin, Bérénice Grec, Francesco Salvarani. The Maxwell-Stefan diffusion limit for a kinetic model of mixtures. Acta Applicandae Mathematicae, 2015, 136 (1), pp.79-90. 10.1007/s10440-0149886-z . hal-00554744v2

\section{HAL Id: hal-00554744 \\ https://hal.science/hal-00554744v2}

Submitted on 21 Nov 2013

HAL is a multi-disciplinary open access archive for the deposit and dissemination of scientific research documents, whether they are published or not. The documents may come from teaching and research institutions in France or abroad, or from public or private research centers.
L'archive ouverte pluridisciplinaire HAL, est destinée au dépôt et à la diffusion de documents scientifiques de niveau recherche, publiés ou non, émanant des établissements d'enseignement et de recherche français ou étrangers, des laboratoires publics ou privés.

\section{(1) (1) $\$$}

Distributed under a Creative Commons Attribution - NonCommercial - NoDerivatives| 4.0 


\title{
THE MAXWELL-STEFAN DIFFUSION LIMIT FOR A KINETIC MODEL OF MIXTURES
}

\author{
LAURENT BOUDIN, BÉRÉNICE GREC, AND FRANCESCO SALVARANI
}

\begin{abstract}
We consider the non-reactive elastic Boltzmann equation for multicomponent gaseous mixtures. We deduce, under the standard diffusive scaling, that well prepared initial conditions lead to solutions satisfying the Maxwell-Stefan diffusion equations in the vanishing Mach and Knudsen numbers limit.
\end{abstract}

\section{INTRODUCTION}

The derivation of macroscopic equations starting from kinetic theory is a very active research field. The interest on such a question has a long history, being even pointed out by Hilbert in his famous lecture [17] delivered at the International Congress of Mathematicians, in Paris in 1900. The problem, known as Hilbert's sixth problem, has been translated in a rigorous mathematical language in a series of pioneering papers $[2,3,4]$. That led to significant articles, such as $[15,16]$, where the authors established a Navier-Stokes limit for the Boltzmann equation considered over $\mathbb{R}^{3}$.

All the aforementioned papers deal with a mono-species, monatomic and ideal gas. However, many common physical situations are more intricate: multi-species mixtures, polyatomic gases, chemical reactions, etc. In this work, we focus on a multi-species mixture of monatomic ideal gases with no chemical reactions.

The mathematical study of Boltzmann-like equations describing such a mixture is far more complex, mainly because of the presence of multi-species kernels, with cross interactions between the different densities describing each component of the mixture. The readers can refer, for example, to $[23,21]$ as founding works on kinetic models for mixtures, to $[13,1,9]$ with a focus on BGK models, to [11] for Boltzmann equations with chemical reactions, and to [7] for a Boltzmann model very similar to the one studied here.

However, the relationship (even at a formal level) between the kinetic level and the macroscopic description is crucial and specifies the range of validity of the target equations.

On the macroscopic point of view, the time evolution of diffusive phenomena for mixtures is well described by the Maxwell-Stefan equations [20, 24] (see [19] for a fairly complete review on the main aspects of multicomponent diffusive phenomena). However, its mathematical study is very recent and solid results on the subject only appeared in the last few years $[14,5,8,18]$. Let us emphasize that this problem has not a mere academical interest, since it has applications to the respiration mechanism $[25,10,6]$, in particular when dealing with a Helium/Oxygen/Carbon dioxide mixture in the lung.

Note that the Maxwell-Stefan equations lie in the class of cross diffusion models, which are commonly introduced in population dynamics, see [22] for instance.

Date: November 13, 2013. 
The relationship between the kinetic description of a phenomena and its macroscopic picture governed by the Maxwell-Stefan equations is still an open question. In this article, we shall show that well prepared initial conditions formally generate, in the diffusive scaling, solutions of the Boltzmann equation for gas mixtures satisfying the Maxwell-Stefan equations in the asymptotic regime.

Our work is organized as follows. First, we briefly recall the Maxwell-Stefan model. Then we propose a Boltzmann-type model of multi-species mixtures and detail the mono and bi-species collision kernels involved in the kinetic equations. Finally, we formally discuss the diffusive asymptotics of our kinetic model towards the Maxwell-Stefan equations.

\section{The Maxwell-Stefan Model}

Consider a bounded domain $\Omega \subset \mathbb{R}^{3}$, with a smooth boundary. We deal with an ideal gas mixture constituted with $I \geq 2$ species with molecular masses $m_{i}$, in a purely diffusive setting (i.e. without any convective effect).

For each species of the mixture $\mathcal{A}_{i}, 1 \leq i \leq I$, we define its concentration $c_{i}$, only depending on the macroscopic variables of time $t \in \mathbb{R}^{+}$and position $x \in \Omega$. We can also define the (diffusive) concentration flux $F_{i}$ of species $\mathcal{A}_{i}$. Both quantities are involved in the continuity equation, holding for any $i$,

$$
\partial_{t} c_{i}+\nabla_{x} \cdot F_{i}=0 \quad \text { on } \mathbb{R}_{+}^{*} \times \Omega .
$$

Let $c=\sum c_{i}$ be the total concentration of the mixture and set $n_{i}=c_{i} / c$ the mole fraction of species $\mathcal{A}_{i}$. The Maxwell-Stefan equations give relationships between the fluxes and the concentrations. They can be written, for any $i$, as

$$
-c \nabla_{x} n_{i}=\frac{1}{c} \sum_{j \neq i} \frac{c_{j} F_{i}-c_{i} F_{j}}{D_{i j}} \quad \text { on } \mathbb{R}_{+}^{*} \times \Omega
$$

where $D_{i j}$ are the so-called effective diffusion coefficients between the species $\mathcal{A}_{i}$ and $\mathcal{A}_{j}$. For obvious physical reasons, the diffusion coefficients are symmetric with respect to the particles exchange, i.e. $D_{i j}=D_{j i}$.

By summing (2) over all $1 \leq i \leq I$, we observe that the Maxwell-Stefan laws are linearly dependent. More precisely, there are exactly $(I-1)$ independent equalities of type $(2)$.

Hence, we need one closure (vectorial) relationship. If one works in a closed system with global constant and uniform temperature and pressure, as in Duncan and Toor's experiment [12], it is natural to assume that there is a transient equimolar diffusion in the mixture before reaching the stationary state. That means that the total diffusive flux satisfies

$$
\sum_{i=1}^{I} F_{i}=0 \quad \text { on } \mathbb{R}_{+}^{*} \times \Omega .
$$

In the whole paper, we focus on an equimolar diffusion process in a closed system and study Equations (1)-(3). Of course, in some other realistic situations, the systems may be not closed, so that the equimolar diffusion assumption does not hold any more (see for instance [10]).

Summing (1) over $i$, one can observe that $c$ does not depend on $t$, and equals its initial value. It is then clear that, if we assume that the molecules of the mixture are initially uniformly distributed, the quantity $c$ does not depend on $x$ either. Note that this assumption prevents vacuum in the mixture. 
We still need a set of boundary conditions to ensure that the system is closed, that is for any $i$,

$$
\nu \cdot F_{i}=0 \text { on } \mathbb{R}_{+} \times \partial \Omega,
$$

where $\nu(x)$ is the normal vector going out from $\Omega$ at $x \in \partial \Omega$.

\section{A Kinetic MODEL FOR GASEOUS MiXTURES}

From now on, we consider a mixture constituted with monatomic ideal gases. The asymptotics is formally investigated in this case only, but we must emphasize that the formal limit should also hold for polyatomic gases.

3.1. Framework. For each species of the mixture $\mathcal{A}_{i}$, we introduce the corresponding distribution function $f_{i}$, which depends on time $t \in \mathbb{R}^{+}$, space position $x \in \Omega$ and velocity $v \in \mathbb{R}^{3}$. For any $i$, $f_{i}(t, x, v) \mathrm{d} x \mathrm{~d} v$ denotes the quantity of matter, expressed in moles, of species $\mathcal{A}_{i}$ in the mixture, at time $t$ in an elementary volume of the space phase of size $\mathrm{d} x \mathrm{~d} v$ centred at $(x, v)$. The distribution function is then related to $c_{i}$ thanks to

$$
c_{i}(t, x)=\int_{\mathbb{R}^{3}} f_{i}(t, x, v) \mathrm{d} v, \quad t \geq 0, \quad x \in \Omega .
$$

Since the mixture is non reactive, only mechanical collisions between molecules are allowed. More precisely, let us consider two molecules of species $\mathcal{A}_{i}$ and $\mathcal{A}_{j}, 1 \leq i, j \leq I$, with respective masses $m_{i}, m_{j}$, and respective pre-collisional velocities $v^{\prime}, v_{*}^{\prime}$. After a collision, the particles belong to the same species, so they still have the same masses, but their velocities have changed and are now denoted by $v$ and $v_{*}$. The collisions are supposed to be elastic. Therefore, both momentum and kinetic energy are conserved:

$$
m_{i} v^{\prime}+m_{j} v_{*}^{\prime}=m_{i} v+m_{j} v_{*}, \quad \frac{1}{2} m_{i}\left|v^{\prime}\right|^{2}+\frac{1}{2} m_{j}\left|v_{*}^{\prime}\right|^{2}=\frac{1}{2} m_{i}|v|^{2}+\frac{1}{2} m_{j}\left|v_{*}\right|^{2} .
$$

From (6), it is possible to write $v^{\prime}$ and $v_{*}^{\prime}$ with respect to $v$ and $v_{*}$ :

$$
v^{\prime}=\frac{1}{m_{i}+m_{j}}\left(m_{i} v+m_{j} v_{*}+m_{j}\left|v-v_{*}\right| \sigma\right), \quad v_{*}^{\prime}=\frac{1}{m_{i}+m_{j}}\left(m_{i} v+m_{j} v_{*}-m_{i}\left|v-v_{*}\right| \sigma\right),
$$

where $\sigma$ is an arbitrary element of $\mathbb{S}^{2}$, which takes into account that (6) allows two degrees of freedom. Note that, if $m_{i}=m_{j}$, we recover the standard collision rules in the Boltzmann equation.

Let us now introduce the collision operators.

3.2. Mono-species collision operators. They allow to recover the standard collision operators in the Boltzmann equation. Let $1 \leq i \leq I$. If $f:=f(v)$ is a nonnegative function, the collision operator describing the interactions between molecules of species $\mathcal{A}_{i}$ is defined by

$$
Q_{i}^{\mathrm{m}}(f, f)(v)=\int_{\mathbb{R}^{3}} \int_{\mathbb{S}^{2}} B_{i}\left(v, v_{*}, \sigma\right)\left[f\left(v^{\prime}\right) f\left(v_{*}^{\prime}\right)-f(v) f\left(v_{*}\right)\right] \mathrm{d} \sigma \mathrm{d} v_{*},
$$

where $v^{\prime}, v_{*}^{\prime}$ are defined by (7) with $m_{i}=m_{j}$, and the cross section $B_{i}$ satisfies the microreversibility assumptions: $B_{i}\left(v, v_{*}, \sigma\right)=B_{i}\left(v_{*}, v, \sigma\right)$ and $B_{i}\left(v, v_{*}, \sigma\right)=B_{i}\left(v^{\prime}, v_{*}^{\prime}, \sigma\right)$. It can also be written under a weak form, for instance,

$$
\begin{aligned}
& \int_{\mathbb{R}^{3}} Q_{i}^{\mathrm{m}}(f, f)(v) \psi(v) \mathrm{d} v= \\
& -\frac{1}{4} \iint_{\mathbb{R}^{6}} \int_{\mathbb{S}^{2}} B_{i}\left(v, v_{*}, \sigma\right)\left[f\left(v^{\prime}\right) f\left(v_{*}^{\prime}\right)-f(v) f\left(v_{*}\right)\right]\left[\psi\left(v^{\prime}\right)+\psi\left(v_{*}^{\prime}\right)-\psi(v)-\psi\left(v_{*}\right)\right] \mathrm{d} \sigma \mathrm{d} v_{*} \mathrm{~d} v,
\end{aligned}
$$


for any $\psi: \mathbb{R}^{3} \rightarrow \mathbb{R}$ such that the first integral in (9) is well defined. Equation (9) is obtained from (8) by using the changes of variables $\left(v, v_{*}\right) \mapsto\left(v_{*}, v\right)$ and $\left(v, v_{*}\right) \mapsto\left(v^{\prime}, v_{*}^{\prime}\right), \sigma \in \mathbb{S}^{2}$ remaining fixed.

These weak forms classically allow to get the conservation, for each species $\mathcal{A}_{i}$, of the total number of molecules, the total momentum and the kinetic energy by successively choosing $\psi(v)=1$, $v$ and $|v|^{2} / 2$.

3.3. Bi-species collision operators. Let $i, j$ such that $1 \leq i, j \leq I$ and $i \neq j$. If $f:=f(v)$ and $g:=g\left(v_{*}\right)$ are nonnegative functions, the collision operator describing the interactions between molecules of species $\mathcal{A}_{i}$ and $\mathcal{A}_{j}$ is defined by

$$
Q_{i j}^{\mathrm{b}}(f, g)(v)=\int_{\mathbb{R}^{3}} \int_{\mathbb{S}^{2}} B_{i j}\left(v, v_{*}, \sigma\right)\left[f\left(v^{\prime}\right) g\left(v_{*}^{\prime}\right)-f(v) g\left(v_{*}\right)\right] \mathrm{d} \sigma \mathrm{d} v_{*},
$$

where $v^{\prime}$ and $v_{*}^{\prime}$, are defined thanks to (7), and the cross section $B_{i j}$ satisfies the microreversibility assumptions $B_{i j}\left(v, v_{*}, \sigma\right)=B_{j i}\left(v_{*}, v, \sigma\right)$ and $B_{i j}\left(v, v_{*}, \sigma\right)=B_{i j}\left(v^{\prime}, v_{*}^{\prime}, \sigma\right)$. In the same way as in the mono-species case, there are several weak formulations involving $Q_{i j}^{\mathrm{b}}$, for instance,

$$
\begin{aligned}
& \int_{\mathbb{R}^{3}} Q_{i j}^{\mathrm{b}}(f, g)(v) \psi(v) \mathrm{d} v \\
&=-\frac{1}{2} \iint_{\mathbb{R}^{6}} \int_{\mathbb{S}^{2}} B_{i j}\left(v, v_{*}, \sigma\right) {\left[f\left(v^{\prime}\right) g\left(v_{*}^{\prime}\right)-f(v) g\left(v_{*}\right)\right]\left[\psi\left(v^{\prime}\right)-\psi(v)\right] \mathrm{d} \sigma \mathrm{d} v \mathrm{~d} v_{*} } \\
&=\iint_{\mathbb{R}^{6}} \int_{\mathbb{S}^{2}} B_{i j}\left(v, v_{*}, \sigma\right) f(v) g\left(v_{*}\right)\left[\psi\left(v^{\prime}\right)-\psi(v)\right] \mathrm{d} \sigma \mathrm{d} v \mathrm{~d} v_{*},
\end{aligned}
$$

or

$$
\begin{aligned}
& \int_{\mathbb{R}^{3}} Q_{i j}^{\mathrm{b}}(f, g)(v) \psi(v) \mathrm{d} v+\int_{\mathbb{R}^{3}} Q_{j i}^{\mathrm{b}}(g, f)(v) \phi(v) \mathrm{d} v= \\
& -\frac{1}{2} \iint_{\mathbb{R}^{6}} \int_{\mathbb{S}^{2}} B_{i j}\left(v, v_{*}, \sigma\right)\left[f\left(v^{\prime}\right) g\left(v_{*}^{\prime}\right)-f(v) g\left(v_{*}\right)\right]\left[\psi\left(v^{\prime}\right)+\phi\left(v_{*}^{\prime}\right)-\psi(v)-\phi\left(v_{*}\right)\right] \mathrm{d} \sigma \mathrm{d} v \mathrm{~d} v_{*},
\end{aligned}
$$

for any $\psi, \phi: \mathbb{R}^{3} \rightarrow \mathbb{R}$ such that the first integrals in (11)-(12) are well defined. Let us emphasize that $\left(v, v^{\prime}\right)$ and $\left(v_{*}, v_{*}^{\prime}\right)$ are respectively associated to species $\mathcal{A}_{i}$ and $\mathcal{A}_{j}$. Equations (11)-(12) are obtained from (10) by using the same changes of variables $\left(v, v_{*}\right) \mapsto\left(v_{*}, v\right)$ and $\left(v, v_{*}\right) \mapsto\left(v^{\prime}, v_{*}^{\prime}\right)$, as in the mono-species case.

The choice $\psi(v)=1$ in (11) allows to recover the conservation of the total number of molecules of species $\mathcal{A}_{i}$. Moreover, if we set $\psi(v)=m_{i} v$ and $\phi\left(v_{*}\right)=m_{j} v_{*}$, and then $\psi(v)=m_{i}|v|^{2} / 2$ and $\phi(v)=m_{j}\left|v_{*}\right|^{2} / 2$, and plug those values in (12), we recover the conservation of the momentum and kinetic energy when species $\mathcal{A}_{i}$ and $\mathcal{A}_{j}$ are simultaneously considered, i.e.

$$
\int_{\mathbb{R}^{3}} Q_{i j}^{\mathrm{b}}(f, g)(v)\left(\begin{array}{c}
m_{i} v \\
m_{i}|v|^{2} / 2
\end{array}\right) \mathrm{d} v+\int_{\mathbb{R}^{3}} Q_{j i}^{\mathrm{b}}(g, f)(v)\left(\begin{array}{c}
m_{j} v \\
m_{j}|v|^{2} / 2
\end{array}\right) \mathrm{d} v=0 .
$$

3.4. Boltzmann's equations. The system of coupled equations satisfied by the set of unknowns $\left(f_{i}\right)_{1 \leq i \leq I}$ is hence

$$
\partial_{t} f_{i}+v \cdot \nabla_{x} f_{i}=Q_{i}^{\mathrm{m}}\left(f_{i}, f_{i}\right)+\sum_{j \neq i} Q_{i j}^{\mathrm{b}}\left(f_{i}, f_{j}\right) \quad \text { on } \mathbb{R}_{+}^{*} \times \Omega \times \mathbb{R}^{3} .
$$


The conservation laws of mass, momentum and kinetic energy are guaranteed by the weak forms (9) and (12). The boundary conditions are not detailed here but they are chosen as specular reflections on $\partial \Omega$ to fit the macroscopic boundary conditions (4).

\section{The Maxwell-Stefan diffusion limit}

The Maxwell-Stefan equations describe a purely diffusive behaviour. Therefore, we cannot hope to deduce them only by scaling a kinetic model that, in principle, can describe also convection phenomena, without making any additional assumptions on the time evolution of the system and on the initial conditions.

In order to study the relationships between the kinetic system and the Maxwell-Stefan equations, we need to clearly identify the physical situation that leads to the Maxwell-Stefan cross diffusion phenomenon and impose to the kinetic system the same physical properties. We hence assume that the scaling on the kinetic equation allows the description of diffusion phenomena, i.e. we suppose that the Knudsen and the Mach numbers are of the same order of magnitude and that they can be considered very small. Moreover, we assume that

- the gaseous mixture is dense and has reached its global mechanical equilibrium;

- there exists a uniform (in space) and constant (in time) temperature $T>0$;

- the bulk velocity of the initial condition is small and it goes to zero in the vanishing Knudsen and Mach numbers limit.

We remark, however, that the mixture may be initially non-homogeneous in space, i.e. the system is far from its macroscopic equilibrium.

4.1. Scaled equation. From now on, let us focus on the Maxwell molecules case. It means that each cross section $B_{i j}$ depends on $v, v_{*}$ and $\sigma$ only through the deviation angle $\theta \in[0, \pi]$ between $v-v_{*}$ and $\sigma$, and more precisely through its cosine. For each $(i, j)$ with $i \neq j$, there exists a function $b_{i j}:[-1,1] \rightarrow \mathbb{R}_{+}$such that

$$
B_{i j}\left(v, v_{*}, \sigma\right)=b_{i j}\left(\frac{v-v_{*}}{\left|v-v_{*}\right|} \cdot \sigma\right)=b_{i j}(\cos \theta) .
$$

We moreover assume that $b_{i j}$ is even and that $b_{i j} \in L^{1}(-1,1)$, following Grad's angular cutoff assumption. Thanks to the microreversibility assumption and because of the parity of $b_{j i}$, we note that

$$
\begin{aligned}
b_{i j}(\cos \theta)=b_{i j}\left(\frac{v-v_{*}}{\left|v-v_{*}\right|} \cdot \sigma\right)=B_{i j}\left(v, v_{*}, \sigma\right) & =B_{j i}\left(v_{*}, v, \sigma\right) \\
& =b_{j i}\left(\frac{v_{*}-v}{\left|v-v_{*}\right|} \cdot \sigma\right)=b_{j i}\left(\frac{v-v_{*}}{\left|v-v_{*}\right|} \cdot \sigma\right)=b_{j i}(\cos \theta),
\end{aligned}
$$

which ensures $b_{i j}=b_{j i}$.

We here make no formal assumption about the mono-species cross section $B_{i}$, but it seems logical that each $B_{i}$ satisfies the same properties as the bi-species cross sections.

For the readers' sake, let us introduce in Figure 1 the other angular variable $\varphi \in[0,2 \pi]$, so that we can write the Euclidean coordinates of $\sigma$ with respect to $\theta$ and $\varphi$, i.e.

$$
\sigma_{(1)}=\sin \theta \cos \varphi, \quad \sigma_{(2)}=\sin \theta \sin \varphi, \quad \sigma_{(3)}=\cos \theta .
$$




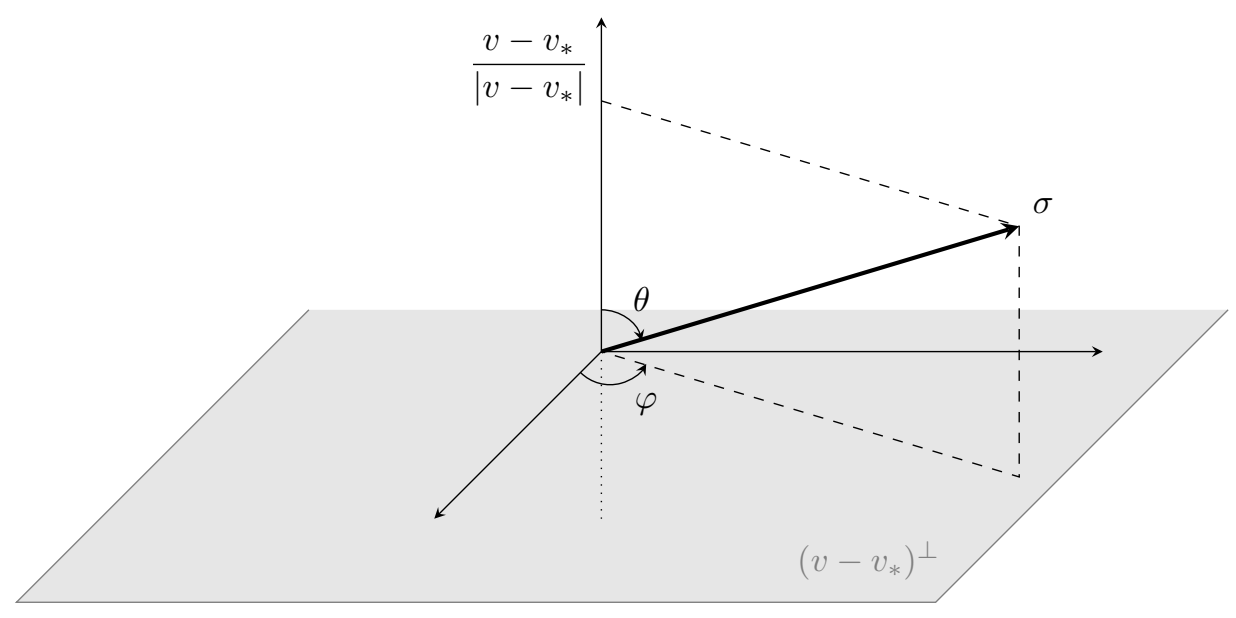

Figure 1. Angular variables to describe $\sigma \in \mathbb{S}^{2}$

In the classical diffusive limit, the scaling is held by the mean free path $\varepsilon>0$ and the corresponding unknowns in this regime are denoted $\left(f_{i}^{\varepsilon}\right)_{1 \leq i \leq I}$. Each distribution function $f_{i}^{\varepsilon}$ hence solves the scaled version of (14), that is

$$
\varepsilon \partial_{t} f_{i}^{\varepsilon}+v \cdot \nabla_{x} f_{i}^{\varepsilon}=\frac{1}{\varepsilon} Q_{i}^{\mathrm{m}}\left(f_{i}^{\varepsilon}, f_{i}^{\varepsilon}\right)+\frac{1}{\varepsilon} \sum_{j \neq i} Q_{i j}^{\mathrm{b}}\left(f_{i}^{\varepsilon}, f_{j}^{\varepsilon}\right), \quad \text { on } \mathbb{R}_{+}^{*} \times \Omega \times \mathbb{R}^{3} .
$$

Finally, we define $\left(c_{i}^{\varepsilon}\right)_{1 \leq i \leq I}$ through (5), for each distribution function $f_{i}^{\varepsilon}$ :

$$
c_{i}^{\varepsilon}(t, x)=\int_{\mathbb{R}^{3}} f_{i}^{\varepsilon}(t, x, v) \mathrm{d} v, \quad t \geq 0, \quad x \in \Omega .
$$

4.2. Ansatz. We assume that the initial conditions of the system of Boltzmann equations (15) are local Maxwellian functions, with small macroscopic velocity (since we are interested in a purely diffusive setting), i.e. the initial conditions have the form

$$
\left(f_{i}^{\text {in }}\right)^{\varepsilon}(x, v)=c_{i}^{\text {in }}(x)\left(\frac{m_{i}}{2 \pi k T}\right)^{3 / 2} e^{-m_{i}\left|v-\varepsilon u_{i}^{\text {in }}(x)\right|^{2} / 2 k T}, \quad x \in \Omega, \quad v \in \mathbb{R}^{3},
$$

where $T>0$ is a fixed constant, and

$$
c_{i}^{\text {in }}: \Omega \rightarrow \mathbb{R}_{+}, \quad u_{i}^{\text {in }}: \Omega \rightarrow \mathbb{R}^{3}, \quad 1 \leq i \leq I,
$$

do not depend on $\varepsilon$. We moreover suppose that

$$
\sum_{i=1}^{I} c_{i}^{\text {in }}=1 \quad \text { on } \Omega
$$

Since each $\left(f_{i}^{\text {in }}\right)^{\varepsilon}$ has the form (16), we immediately have, for any $i$,

$$
\frac{1}{\varepsilon} \int_{\mathbb{R}^{3}} v\left(f_{i}^{\text {in }}\right)^{\varepsilon}(x, v) \mathrm{d} v=c_{i}^{\text {in }}(x) u_{i}^{\text {in }}(x), \quad x \in \Omega .
$$


We assume that the system evolution leaves the distribution functions in the local Maxwellian state, with the same constant and homogeneous temperature $T$. We hence suppose that there exist

$$
c_{i}^{\varepsilon}: \mathbb{R}_{+} \times \Omega \rightarrow \mathbb{R}_{+}, \quad u_{i}^{\varepsilon}: \mathbb{R}_{+} \times \Omega \rightarrow \mathbb{R}^{3}, \quad 1 \leq i \leq I,
$$

such that

$$
f_{i}^{\varepsilon}(t, x, v)=c_{i}^{\varepsilon}(t, x)\left(\frac{m_{i}}{2 \pi k T}\right)^{3 / 2} e^{-m_{i}\left|v-\varepsilon u_{i}^{\varepsilon}(t, x)\right|^{2} / 2 k T}, \quad t>0, \quad x \in \Omega, \quad v \in \mathbb{R}^{3} .
$$

Since $T$ is constant, the macroscopic equations should be obtained only through the conservation laws of mass and momentum. The moments of order 0 and 1 of each distribution function can be computed thanks to Ansatz (17):

$$
\int_{\mathbb{R}^{3}} f_{i}^{\varepsilon}(t, x, v)\left(\begin{array}{c}
1 \\
v
\end{array}\right) \mathrm{d} v=\left(\begin{array}{c}
c_{i}^{\varepsilon}(t, x) \\
\varepsilon c_{i}^{\varepsilon}(t, x) u_{i}^{\varepsilon}(t, x)
\end{array}\right), \quad t>0, \quad x \in \Omega .
$$

Note that the first moment of $f_{i}^{\varepsilon}$ is of order 1 in $\varepsilon$ since we focus on the diffusive asymptotics.

4.2.1. Matter conservation. We first consider the moment of order 0 of the distribution functions. More precisely, for any $i$, we integrate (15) with respect to $v$ in $\mathbb{R}^{3}$, and obtain, thanks to the conservation properties of the collisional operators,

$$
\varepsilon \partial_{t}\left(\int_{\mathbb{R}^{3}} f_{i}^{\varepsilon}(t, x, v) \mathrm{d} v\right)+\nabla_{x} \cdot\left(\int_{\mathbb{R}^{3}} f_{i}^{\varepsilon}(t, x, v) v \mathrm{~d} v\right)=0 .
$$

Using (18), we get, for all $1 \leq i \leq I$,

$$
\partial_{t} c_{i}^{\varepsilon}+\nabla_{x} \cdot\left(c_{i}^{\varepsilon} u_{i}^{\varepsilon}\right)=0 .
$$

4.2.2. Balance of momentum. For $\ell \in\{1,2,3\}$, denote $w_{(\ell)}$ the $\ell$-th component of any vector $w \in \mathbb{R}^{3}$. The balance law of momentum for a given species $\mathcal{A}_{i}$ is obtained by multiplying (15) by $v_{(\ell)}$ and integrating with respect to $v$ in $\mathbb{R}^{3}$. We obtain, for any $i$ and $\ell$,

$$
(20) \varepsilon \partial_{t}\left(\int_{\mathbb{R}^{3}} v_{(\ell)} f_{i}^{\varepsilon}(v) \mathrm{d} v\right)+\nabla_{x} \cdot\left(\int_{\mathbb{R}^{3}} v_{(\ell)} f_{i}^{\varepsilon}(v) v \mathrm{~d} v\right)=\frac{1}{\varepsilon} \sum_{j \neq i} \int_{\mathbb{R}^{3}} v_{(\ell)} Q_{i j}^{\mathrm{b}}\left(f_{i}^{\varepsilon}, f_{j}^{\varepsilon}\right)(v) \mathrm{d} v:=\Theta_{(\ell)}^{\varepsilon},
$$

because the term involving $Q_{i}^{\mathrm{m}}$ vanishes. Let us first focus on $\Theta_{(\ell)}^{\varepsilon}$, which depends on the set of independent variables $(t, x)$. Thanks to $(7)$ and $(11)$ with $\psi(v)=v$, we can write

$$
\begin{aligned}
& \Theta_{(\ell)}^{\varepsilon}=\frac{1}{\varepsilon} \sum_{j \neq i} \iint_{\mathbb{R}^{6}} \int_{\mathbb{S}^{2}} b_{i j}\left(\frac{v-v_{*}}{\left|v-v_{*}\right|} \cdot \sigma\right) f_{i}^{\varepsilon}(v) f_{j}^{\varepsilon}\left(v_{*}\right)\left(v_{(\ell)}^{\prime}-v_{(\ell)}\right) \mathrm{d} \sigma \mathrm{d} v_{*} \mathrm{~d} v \\
& =\frac{1}{\varepsilon} \sum_{j \neq i} \frac{m_{j}}{m_{i}+m_{j}} \iint_{\mathbb{R}^{6}} \int_{\mathbb{S}^{2}} b_{i j}\left(\frac{v-v_{*}}{\left|v-v_{*}\right|} \cdot \sigma\right) f_{i}^{\varepsilon}(v) f_{j}^{\varepsilon}\left(v_{*}\right)\left(v_{*(\ell)}-v_{(\ell)}+\left|v-v_{*}\right| \sigma_{(\ell)}\right) \mathrm{d} \sigma \mathrm{d} v_{*} \mathrm{~d} v .
\end{aligned}
$$

In the previous equality, the term containing $\sigma_{(\ell)}$ vanishes, because of the symmetry properties of $B_{i j}$ with respect to $\sigma$. Indeed, both terms for $\ell=1$ or 2 are zero because

$$
\int_{0}^{2 \pi} \sin \varphi \mathrm{d} \varphi=\int_{0}^{2 \pi} \cos \varphi \mathrm{d} \varphi=0,
$$


and the third one writes

$$
\int_{\mathbb{S}^{2}} b_{i j}\left(\frac{v-v_{*}}{\left|v-v_{*}\right|} \cdot \sigma\right) \sigma_{(3)} \mathrm{d} \sigma=2 \pi \int_{0}^{\pi} \sin \theta \cos \theta b_{i j}(\cos \theta) \mathrm{d} \theta=2 \pi \int_{-1}^{1} \eta b_{i j}(\eta) \mathrm{d} \eta=0,
$$

because $b_{i j}$ is even.

The remaining part of the expression of $\Theta^{\varepsilon}$ can then be written in terms of macroscopic quantities:

$$
\Theta^{\varepsilon}=\varepsilon \sum_{j \neq i} \frac{2 \pi m_{j}\left\|b_{i j}\right\|_{L^{1}}}{m_{i}+m_{j}}\left(c_{i}^{\varepsilon} c_{j}^{\varepsilon} u_{j}^{\varepsilon}-c_{j}^{\varepsilon} c_{i}^{\varepsilon} u_{i}^{\varepsilon}\right) .
$$

The time derivative in (20) can be evaluated by means of (18), so that (20) eventually becomes, for any $\ell \in\{1,2,3\}$ and any $i$,

$$
\varepsilon^{2} \partial_{t}\left(c_{i}^{\varepsilon}\left(u_{i}^{\varepsilon}\right)_{(\ell)}\right)+\nabla_{x} \cdot\left(\int_{\mathbb{R}^{3}} v_{(\ell)} f_{i}^{\varepsilon}(v) v \mathrm{~d} v\right)=\sum_{j \neq i} \frac{2 \pi m_{j}\left\|b_{i j}\right\|_{L^{1}}}{m_{i}+m_{j}}\left(c_{i}^{\varepsilon} c_{j}^{\varepsilon}\left(u_{j}^{\varepsilon}\right)_{(\ell)}-c_{j}^{\varepsilon} c_{i}^{\varepsilon}\left(u_{i}^{\varepsilon}\right)_{(\ell)}\right) .
$$

Let us now focus on the divergence term in (21). We successively write

$$
\begin{gathered}
\nabla_{x} \cdot\left(\int_{\mathbb{R}^{3}} v_{(\ell)} f_{i}^{\varepsilon}(v) v \mathrm{~d} v\right)=\sum_{k=1}^{3} \frac{\partial}{\partial x_{(k)}} \int_{\mathbb{R}^{3}} v_{(\ell)} v_{(k)} f_{i}^{\varepsilon}(v) \mathrm{d} v \\
=\sum_{k=1}^{3} \frac{\partial}{\partial x_{(k)}} \int_{\mathbb{R}^{3}} c_{i}^{\varepsilon}\left(v_{(\ell)}+\varepsilon\left(u_{i}\right)_{(\ell)}^{\varepsilon}\right)\left(v_{(k)}+\varepsilon\left(u_{i}\right)_{(k)}^{\varepsilon}\right)\left(\frac{m_{i}}{2 \pi k T}\right)^{3 / 2} e^{-m_{i}|v|^{2} / 2 k T} \mathrm{~d} v \\
=\sum_{k=1}^{3} \frac{\partial}{\partial x_{(k)}} \int_{\mathbb{R}^{3}} c_{i}^{\varepsilon}\left[\varepsilon^{2}\left(u_{i}^{\varepsilon}\right)_{(\ell)}\left(u_{i}^{\varepsilon}\right)_{(k)}+v_{(\ell)}^{2} \delta_{k \ell}\right]\left(\frac{m_{i}}{2 \pi k T}\right)^{3 / 2} e^{-m_{i}|v|^{2} / 2 k T} \mathrm{~d} v \\
=\varepsilon^{2} \sum_{k=1}^{3} \frac{\partial}{\partial x_{(k)}}\left[c_{i}^{\varepsilon}\left(u_{i}^{\varepsilon}\right)_{(\ell)}\left(u_{i}^{\varepsilon}\right)_{(k)}\right]+\frac{k T}{m_{i}} \frac{\partial c_{i}^{\varepsilon}}{\partial x_{(\ell)}} .
\end{gathered}
$$

We finally obtain, from (21) and the previous equality,

$$
\varepsilon^{2}\left[\partial_{t}\left(c_{i}^{\varepsilon} u_{i}^{\varepsilon}\right)+\nabla_{x} \cdot\left(c_{i}^{\varepsilon} u_{i}^{\varepsilon} \otimes u_{i}^{\varepsilon}\right)\right]+\frac{k T}{m_{i}} \nabla_{x} c_{i}^{\varepsilon}=\sum_{j \neq i} \frac{2 \pi m_{j}\left\|b_{i j}\right\|_{L^{1}}}{m_{i}+m_{j}}\left(c_{i}^{\varepsilon} c_{j}^{\varepsilon} u_{j}^{\varepsilon}-c_{j}^{\varepsilon} c_{i}^{\varepsilon} u_{i}^{\varepsilon}\right)
$$

4.2.3. Macroscopic equations and formal asymptotics. By putting together (19) and (22), we deduce that the Maxwellians (17) are solution of the initial-boundary value problem for the system of scaled Boltzmann equations (15) if $\left(c_{i}^{\varepsilon}, u_{i}^{\varepsilon}\right)$ solves

$$
\begin{aligned}
& \partial_{t} c_{i}^{\varepsilon}+\nabla_{x} \cdot\left(c_{i}^{\varepsilon} u_{i}^{\varepsilon}\right)=0, \\
& \varepsilon^{2} \frac{m_{i}}{k T}\left[\partial_{t}\left(c_{i}^{\varepsilon} u_{i}^{\varepsilon}\right)+\nabla_{x} \cdot\left(c_{i}^{\varepsilon} u_{i}^{\varepsilon} \otimes u_{i}^{\varepsilon}\right)\right]+\nabla_{x} c_{i}^{\varepsilon}=\sum_{j \neq i} \frac{c_{i}^{\varepsilon} c_{j}^{\varepsilon} u_{j}^{\varepsilon}-c_{j}^{\varepsilon} c_{i}^{\varepsilon} u_{i}^{\varepsilon}}{\Delta_{i j}},
\end{aligned}
$$

where

$$
\Delta_{i j}=\frac{2 \pi\left(m_{i}+m_{j}\right) k T}{m_{i} m_{j}\left\|b_{i j}\right\|_{L^{1}}}
$$

Note that the previous coefficients are symmetric with respect to each pair of species since $b_{i j}=b_{j i}$. 
In the following, let us set

$$
F_{i}^{\varepsilon}(t, x)=\frac{1}{\varepsilon} \int_{\mathbb{R}^{3}} v f_{i}^{\varepsilon}(t, x, v) \mathrm{d} v=c_{i}^{\varepsilon}(t, x) u_{i}^{\varepsilon}(t, x), \quad t \geq 0, \quad x \in \Omega,
$$

and denote, as usual when dealing with formal diffusive limits, for any $t \geq 0$ and $x \in \Omega$,

$$
c_{i}(t, x)=\lim _{\varepsilon \rightarrow 0^{+}} c_{i}^{\varepsilon}(t, x), \quad F_{i}(t, x)=\lim _{\varepsilon \rightarrow 0^{+}} F_{i}^{\varepsilon}(t, x) .
$$

Hence, in the limit, Equations (19)-(22) give a system of equations, which has the following form for the density-flux set of unknown $\left(c_{i}, F_{i}\right)$ :

$$
\begin{aligned}
& \partial_{t} c_{i}+\nabla_{x} \cdot F_{i}=0, \\
& -\nabla_{x} c_{i}=\sum_{j \neq i} \frac{c_{j} F_{i}-c_{i} F_{j}}{\Delta_{i j}} .
\end{aligned}
$$

In order to recover the the Maxwell-Stefan system, we still have to prove that $c$ is constant in the limit.

Let us now write the kinetic energy conservation of the whole system before the asymptotics, at the kinetic level. We first observe that

$$
\int_{\mathbb{R}^{3}}|v|^{2} f_{i}^{\varepsilon}(v) \mathrm{d} v=3 \frac{k T}{m_{i}} c_{i}^{\varepsilon}+o(\varepsilon), \quad \int_{\mathbb{R}^{3}}|v|^{2} v f_{i}^{\varepsilon}(v) \mathrm{d} v=5 \varepsilon \frac{k T}{m_{i}} c_{i}^{\varepsilon} u_{i}^{\varepsilon}+o(\varepsilon) .
$$

Then we multiply (15) by $m_{i}|v|^{2} / 2$, integrate with respect to $v \in \mathbb{R}^{3}$ and sum over $i$, to obtain, thanks to (9), (13) and (26),

$$
3 \partial_{t}\left(\sum_{i=1}^{I} c_{i}^{\varepsilon}\right)+5 \nabla_{x} \cdot\left(\sum_{i=1}^{I} c_{i}^{\varepsilon} u_{i}^{\varepsilon}\right)=o(1),
$$

by keeping the lowest order term in $\varepsilon$. We formally perform the asymptotics $\varepsilon \rightarrow 0$ in (27) to simultaneously obtain, thanks to (19),

$$
\partial_{t} c=0 \quad \text { and } \quad \nabla_{x} \cdot\left(\sum_{i=1}^{I} F_{i}\right)=0 .
$$

The second equality is obviously consistent with the boundary conditions (4) and the closure relationship (3). The first one ensures that $c=\sum c_{i}^{\text {in }}=1$, which allows to recover (2) from (25):

$$
\left\{\begin{array}{l}
\partial_{t} c_{i}+\nabla_{x} \cdot F_{i}=0 \text { on } \mathbb{R}_{+}^{*} \times \Omega, \\
-c \nabla_{x} n_{i}=\frac{1}{c} \sum_{j \neq i} \frac{c_{j} F_{i}-c_{i} F_{j}}{D_{i j}} \text { on } \mathbb{R}_{+}^{*} \times \Omega,
\end{array}\right.
$$

where $D_{i j}=\Delta_{i j} / c$ and has the physical dimension of a drag coefficient $\left(\mathrm{m}^{2} \mathrm{~s}^{-1}\right)$.

It is worth noting that, whereas the Euler system for mixtures (23)-(24) is composed of $4 I$ scalar independent equations governing $4 I$ scalar unknown functions, the Maxwell-Stefan system is not closed, since it consists only of $(4 I-3)$ scalar independent equations, as discussed in Section 2 . It is consistent with the limiting procedure: we perform a singular perturbation limit which destroys the main part of the differential operator appearing in the momentum conservation equations. 
Acknowledgements. This work was partially funded by the ANR-08-JCJC-013-01 project M3RS, headed by Céline Grandmont, and by the ANR-11-TECS-0006 project OxHelease, coordinated by Caroline Majoral. BG and FS also want to acknowledge the Reo project-team from Inria Paris-Rocquencourt, for its hospitality which allowed to carry out this article.

\section{REFERENCES}

[1] P. Andries, K. Aoki, and B. Perthame. A consistent BGK-type model for gas mixtures. J. Statist. Phys., 106(56):993-1018, 2002.

[2] C. Bardos, F. Golse, and C.D. Levermore. Sur les limites asymptotiques de la théorie cinétique conduisant à la dynamique des fluides incompressibles. C. R. Acad. Sci. Paris Sér. I Math., 309(11):727-732, 1989.

[3] C. Bardos, F. Golse, and C.D. Levermore. Fluid dynamic limits of kinetic equations. I. Formal derivations. $J$. Statist. Phys., 63(1-2):323-344, 1991.

[4] C. Bardos, F. Golse, and C.D. Levermore. Fluid dynamic limits of kinetic equations. II. Convergence proofs for the Boltzmann equation. Comm. Pure Appl. Math., 46(5):667-753, 1993.

[5] D. Bothe. On the Maxwell-Stefan approach to multicomponent diffusion. In Parabolic problems, volume 80 of Progr. Nonlinear Differential Equations Appl., pages 81-93. Birkhäuser/Springer Basel AG, Basel, 2011.

[6] L. Boudin, D. Götz, and B. Grec. Diffusion models of multicomponent mixtures in the lung. In CEMRACS 2009: Mathematical modelling in medicine, volume 30 of ESAIM Proc., pages 90-103. EDP Sci., Les Ulis, 2010.

[7] L. Boudin, B. Grec, M. Pavić, and F. Salvarani. Diffusion asymptotics of a kinetic model for gaseous mixtures. Kinet. Relat. Models, 6(1):137-157, 2013.

[8] L. Boudin, B. Grec, and F. Salvarani. A mathematical and numerical analysis of the Maxwell-Stefan diffusion equations. Discrete Contin. Dyn. Syst. Ser. B, 17(5):1427-1440, 2012.

[9] S. Brull, V. Pavan, and J. Schneider. Derivation of a BGK model for mixtures. Eur. J. Mech. B Fluids, 33:74-86, 2012.

[10] H. K. Chang. Multicomponent diffusion in the lung. Fed. Proc., 39(10):2759-2764, 1980.

[11] L. Desvillettes, R. Monaco, and F. Salvarani. A kinetic model allowing to obtain the energy law of polytropic gases in the presence of chemical reactions. Eur. J. Mech. B Fluids, 24(2):219-236, 2005.

[12] J. B. Duncan and H. L. Toor. An experimental study of three component gas diffusion. AIChE Journal, 8(1):3841, 1962.

[13] V. Garzó, A. Santos, and J. J. Brey. A kinetic model for a multicomponent gas. Phys. Fluids A, 1(2):380-383, 1989.

[14] V. Giovangigli. Multicomponent flow modeling. Modeling and Simulation in Science, Engineering and Technology. Birkhäuser Boston Inc., Boston, MA, 1999.

[15] F. Golse and L. Saint-Raymond. The Navier-Stokes limit of the Boltzmann equation for bounded collision kernels. Invent. Math., 155(1):81-161, 2004.

[16] F. Golse and L. Saint-Raymond. The incompressible Navier-Stokes limit of the Boltzmann equation for hard cutoff potentials. J. Math. Pures Appl. (9), 91(5):508-552, 2009.

[17] D. Hilbert. Mathematical problems. Bull. Amer. Math. Soc., 8(10):437-479, 1902.

[18] A. Jüngel and I. V. Stelzer. Existence analysis of Maxwell-Stefan systems for multicomponent mixtures. SIAM J. Math. Anal., 45(4):2421-2440, 2013.

[19] R. Krishna and J. A. Wesselingh. The Maxwell-Stefan approach to mass transfer. Chem. Eng. Sci., 52(6):861911, 1997.

[20] J. C. Maxwell. On the dynamical theory of gases. Phil. Trans. R. Soc., 157:49-88, 1866.

[21] T. F. Morse. Kinetic model equations for a gas mixture. Phys. Fluids, 7:2012-2013, 1964.

[22] N. Shigesada, K. Kawasaki, and E. Teramoto. Spatial segregation of interacting species. J. Theoret. Biol., $79(1): 83-99,1979$.

[23] L. Sirovich. Kinetic modeling of gas mixtures. Phys. Fluids, 5:908-918, 1962.

[24] J. Stefan. Ueber das Gleichgewicht und die Bewegung insbesondere die Diffusion von Gasgemengen. Akad. Wiss. Wien, 63:63-124, 1871.

[25] M. Thiriet, D. Douguet, J.-C. Bonnet, C. Canonne, and C. Hatzfeld. The effect on gas mixing of a He- $\mathrm{O}_{2}$ mixture in chronic obstructive lung diseases. Bull. Eur. Physiopathol. Respir., 15(5):1053-1068, 1979. In French. 
L.B.: UPMC Univ Paris 06, UMR 7598 LJll, Paris, F-75005, France \& Inria Paris-Rocquencourt, Reo Project-team, BP 105, F-78153 Le Chesnay Cedex, France

E-mail address: laurent.boudin@upmc.fr

B.G.: MAP5, CNRS UMR 8145, Université Paris Descartes, F-75006 Paris, France

E-mail address: berenice.grec@parisdescartes.fr

F.S.: Dipartimento di Matematica, Università degli Studi di Pavia, Via Ferrata 1 - I-27100 Pavia, ITALY

E-mail address: francesco.salvarani@unipv.it 